\title{
SYNTHESIS, DFT, SPECTROSCOPIC CHARACTERISATION (FT-IR, FT-RAMAN, NMR AND UV-VISIBLE) AND MOLECULAR DOCKING INVESTIGATION OF 3-(4- BROMOPHENYL)-1-(THIOPHEN-2-YL) PROP-2-EN-1 ONE
}

\author{
P. Rajamani and N. Sundaraganesan* \\ ${ }^{1}$ Department of Physics, Faculty of Engineering and Technology, Annamalai University, \\ Annamalai Nagar, 608002 Chidambaram, Tamil Nadu, India. \\ *E-mail: Sundaraganesan_n@yahoo.com
}

\begin{abstract}
3-(4-bromophenyl)-1-(thiophene-2-yl) prop-2-en-1 one (BPTP) was synthesized and characterized using FT-IR (3500-500 $\left.\mathrm{cm}^{-1}\right)$, FT-Raman (3500-50 $\left.\mathrm{cm}^{-1}\right)$ and UV-Visible spectrum $(200-800 \mathrm{~nm})$ to confirmed the synthesized molecule structure. Molecular geometry and vibrational wavenumbers of BPTP were studied by using B3LYP/6$311 \mathrm{G}(\mathrm{d}, \mathrm{p})$ approach. The Total energy distribution (TED) utilized the VEDA 4 program for a complete vibrational analysis. The proton and carbon nuclear magnetic resonance (NMR) spectrum chemical shifts of the BPTP target compound were theoretically carried out by the gauge independent atomic orbital (GIAO) technique and were compared with the experimental solutions. The title molecule geometrical parameter was the best assent with the experimental one. NBO investigation, the conversion in the electron density of anti-bonding orbital and stabilization energies have been calculated to stretch clear proof of stabilization in the hyperconjugation of hydrogen-bonded interactions. NMR techniques of the target molecule are explained both experimentally and computational. The measured HOMO-LUMO band gap energies confirm that charge transfer occurs within the target compound. The computational technique using the Molecular Electrostatic Potential map (MEP) was also been calculated to stretch nucleophilic and electrophilic nature. The local reactively of the molecule was studied using the Fukui indices. Molecular docking studies were also been followed to understand the BPTP compound biological activities.

Keywords: BPTP, UV-Vis, NMR, HOMO-LUMO, MEP.
\end{abstract}

(C) RASĀYAN. All rights reserved

\section{INTRODUCTION}

The chalcone moiety has a $\pi$-conjugated system that gives an extreme charge-transfer axis. The moiety obeys the applications is used telecommunications, laser, optical computers, data storage, carrying the information optical sensing and optical systems. Chalcone moiety well switching behavior and optical limiting with a high-intensity laser pulse. Chalcone moiety has been used as pharmaceutical activities including antimicrobial (antifungal and antibacterial), anti-inflammatory, antileishmanial, antiviral, antitubercular, antioxidant, anticancer and antimalarial and etc., Recently, Shivaraj $\mathrm{R}$ et al have investigated the harmonic frequencies of (2E)-3-(4-fluorophenyl)-1-(4-\{[(1E)-(4fluorophenyl)methylene]amino phenyl)prop-2-en-1-one computed utilizingB3LYP/6-311G (d,p) method. Similarly, Nathiya A et. al, have reported the computed and experimental solutions on the synthesized compound (E)-N'(thiophene-2yl methylene) isonicotinohydrazide. A. Theresa et al and S. Bharanidhasan et al have reported that the theoretical and experimental comparison of the following compounds, (E)-1-(benzo[d][1,3]dioxol-6-yl)-3-(6-methoxynaphthalen-2-yl)prop-2-en-1-one,(E)-N'(thiophene-2-ylmethylene) icotinohydrazide. Chemical compounds with chalcone derivative fascinate big research interest due to their presence in lots of organic activities. ${ }^{1}$ Chalcone plays the most important role in biochemistry, so it has optical properties together with high extinction coefficients for absorption in the UV and notable nonlinear optical responses. Therefore, nonlinear optical (NLO) materials can be

Rasayan J. Chem., 12(4), 2149-2165(2019)

http://dx.doi.org/10.31788/RJC.2019.1245436

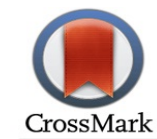


RASĀYAN J. Chem.

Vol. 12 | No. 4 |2149 - 2165| October - December | 2019

stabilized, so that it does not make sense to understand the methods of the chalcone absorption spectrum and electronic transitions. ${ }^{2}$ The best of authors knowledge there was no literature for theoretical investigation of the target compound. This research gap tempted the researchers to taken up the research for experimental and theoretical investigations.

In the present study, the target compound was synthesized and characterized by using analytical methods such as, FT-IR, FT-Raman, NMR, UV-Visible spectroscopy and compared the experimental one. Also, the studies were been carried out on NBO, MEP, Dipole moment, Homo-Lumo and Milliken analysis, etc. Furthermore, the docking analysis was also been carried out to recognize its biological application.

\section{Instruments}

\section{EXPERIMENTAL}

Fourier Transform Infrared range of BPTP transformed into a recorded on a Perkin Elmer FT-IR spectrophotometer using $\mathrm{KBr}$ pellet system as a piece of the area $3500-500 \mathrm{~cm}^{-1}$. Fourier Transform Raman range was recorded on a BRUKER RFS 27 spectrometer utilizing $1064 \mathrm{~nm}$ excitation wavelength from an Nd: YAG Laser inside the region $3500-50 \mathrm{~cm}^{-1}$. The laser yield for this strong example is around $300 \mathrm{~mW}$. The ${ }^{1} \mathrm{H}$ and ${ }^{13} \mathrm{C}$ NMR range become recorded by means of using Bruker AVANCE III $500 \mathrm{MHz}$ (AV 500) spectrometer with the guide of taking $\mathrm{CDCl} 3$ as NMR dissolvable and TMS as reference. UVVisible range of a fluid arrangement of the BPTP is recorded over a scope of $200-800 \mathrm{~nm}$ in the JASCO V-670 PC spectrophotometer, working at goals of $0.5 \mathrm{~nm}$.

\section{Synthesis of 3-(4-bromophenyl)-1-(thiophene-2-yl) prop-2-en-1 One}

A mixture of 4-Bromobenzaldehyde $(0.01 \mathrm{~mL})$ and 2-acetyl thiophene derivatives $(0.01 \mathrm{~mL})$ was dissolved in $10 \mathrm{~mL}$ rectified sprit in a $250 \mathrm{ml}$ conical flask with a magnetic stirrer. Then $10 \mathrm{~mL} \mathrm{NaOH}$ solution was added dropwise to the reaction mixture on stirring for 30 minutes when the solution became turbid. After vigorous stirring for 4-5 hours, the reaction mixture was poured into cold water on filtering off crude chalcone were derived and recrystallized from ethanol.

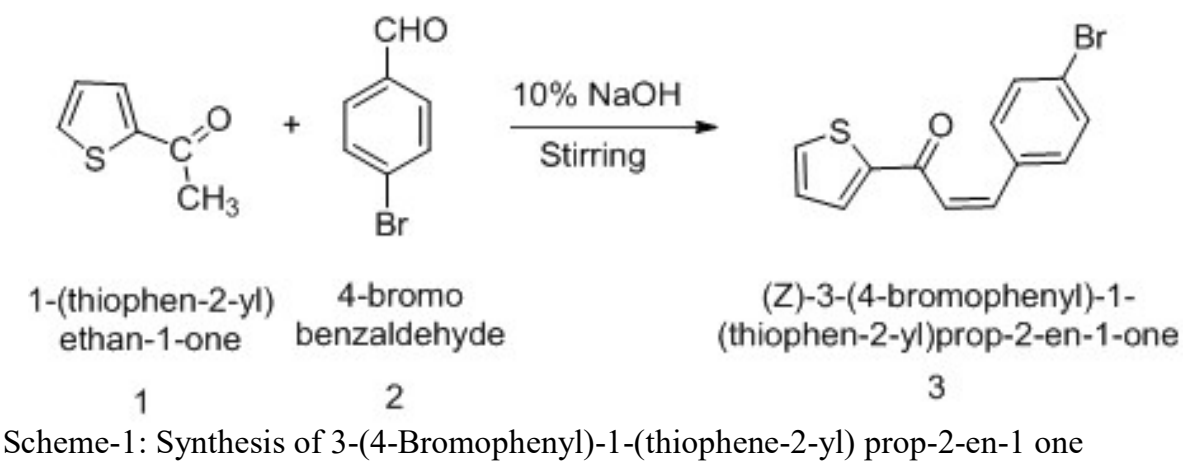

\section{Computational Details}

The computational calculations, BPTP were calculated with the Gaussian $09 \mathrm{~W}$ agendum. ${ }^{3}$ The same techniques are used to calculate the wavenumbers for vibrational modes. Estimated works based on TED has been made in a normal manner. TED were measured using the Vibrational Energy Distribution Analysis (VEDA) agendum of quantum chemical calculated vibration frequencies ${ }^{4}$ graphical interface, optimized molecular systems, vibration frequencies, nuclear systems and bipolar moment, NMR spectrum and UV-Visible spectrum. The proton and carbon nuclear magnetic resonance (NMR) chemical changes BPTP were calculated by the gauge-independent atomic orbital (GIAO) theory in amalgamation with B3LYP/6-311G $(\mathrm{d}, \mathrm{p})$ and related experimental data. ${ }^{5}$

\section{Molecular Geometry}

\section{RESULTS AND DISCUSSION}

The BPTP target compound was optimized by B3LYP/6-311G (d,p) level of basis set. This compound consists of bromophenyl and thiophene ring are fused by chalcone linkage. The calculated bond length of 
RASĀYAN J. Chem.

Vol. 12 | No. 4 |2149 - 2165| October - December | 2019

$\mathrm{C} 2-\mathrm{Br} 25 \rightarrow 1.009$ and $\mathrm{C} 11-\mathrm{S} 15 \rightarrow 1.771$. Since the chalcone moiety is linked with $5 \mathrm{C}$ atom and their corresponding bond length are $\mathrm{C} 5-\mathrm{C} 21\left(1.005 \mathrm{~A}^{\circ}\right), \mathrm{C} 19-\mathrm{O} 22\left(1.258 \mathrm{~A}^{\circ}\right), \mathrm{C} 19-\mathrm{C} 20\left(1.005 \mathrm{~A}^{\circ}\right)$ and $\mathrm{O} 22-\mathrm{C} 21$ $\left(1.355 \mathrm{~A}^{\circ}\right)$. The bond angles of $\mathrm{C} 1-\mathrm{C} 2-\mathrm{Br} 25(120), \mathrm{C} 3-\mathrm{C} 2-\mathrm{Br} 25(120)$ equal value due to attached the bromine atom. C12-C11-S15 (110.5) is expected to be greater than the bond angles S15-C11-C19 (124.7). The dihedral angles of $\mathrm{C} 6-\mathrm{C} 1-\mathrm{C} 2-\mathrm{Br} 25$ (179.9), correspondingly. The optimized structure and geometrical parameters are given in Fig. -1 and Table- $1 .{ }^{6}$

Table -1: Geometrical Parameters [Bond Length $(\AA)$, Bond Angle $\left(^{\circ}\right)$ and Dihedral Angle $\left(^{\circ}\right)$ ] of the BPTP Target Compound.

\begin{tabular}{|c|c|c|c|c|c|}
\hline $\begin{array}{l}\text { Bond } \\
\text { length }\end{array}$ & $\begin{array}{c}\text { B3LYP/61 } \\
3-\mathrm{G}(\mathrm{d}, \mathrm{p})\end{array}$ & Bond angle & $\begin{array}{c}\text { B3LYP/61 } \\
3-\mathrm{G}(\mathrm{d}, \mathrm{p})\end{array}$ & Dihedral angle & $\begin{array}{l}\text { B3LYP/61 } \\
3-\mathrm{G}(\mathrm{d}, \mathrm{p})\end{array}$ \\
\hline $\mathrm{C} 1-\mathrm{C} 2$ & 1.401 & $\mathrm{C} 2-\mathrm{C} 1-\mathrm{C} 6$ & 120 & C6-C1-C2-C3 & 0.0 \\
\hline C1-C6 & 1.401 & $\mathrm{C} 2-\mathrm{C} 2-\mathrm{H} 7$ & 120 & $\mathrm{C} 6-\mathrm{C} 1-\mathrm{C} 2-\mathrm{Br} 25$ & 179.9 \\
\hline $\mathrm{C} 1-\mathrm{H} 7$ & 1.000 & C6-C1-H7 & 120 & $\mathrm{H} 7-\mathrm{C} 1-\mathrm{C} 2-\mathrm{C} 3$ & -180.0 \\
\hline $\mathrm{C} 2-\mathrm{C} 3$ & 1.401 & $\mathrm{C} 1-\mathrm{C} 2-\mathrm{C} 3$ & 120 & $\mathrm{H} 7-\mathrm{C} 1-\mathrm{C} 2-\mathrm{Br} 25$ & 0.0 \\
\hline $\mathrm{C} 2-\mathrm{Br} 25$ & 1.009 & $\mathrm{C} 1-\mathrm{C} 2-\mathrm{Br} 25$ & 120 & $\mathrm{C} 2-\mathrm{C} 1-\mathrm{C} 6-\mathrm{C} 5$ & 0.0 \\
\hline $\mathrm{C} 3-\mathrm{C} 4$ & 1.401 & $\mathrm{C} 3-\mathrm{C} 2-\mathrm{Br} 25$ & 120 & $\mathrm{C} 2-\mathrm{C} 1-\mathrm{C} 6-\mathrm{H} 10$ & 180.0 \\
\hline $\mathrm{C} 3-\mathrm{H} 8$ & 1.000 & $\mathrm{C} 2-\mathrm{C} 3-\mathrm{C} 4$ & 120 & $\mathrm{H} 7-\mathrm{C} 1-\mathrm{C} 6-\mathrm{C} 5$ & 180.0 \\
\hline C4-C5 & 1.401 & C2-C3-H8 & 120 & H7-C1-C6-10 & 0.0 \\
\hline C4-H9 & 1.000 & C2-C3-H8 & 120 & C1-C2-C3-C4 & 0.0 \\
\hline C5-C6 & 1.401 & C3-C4-C5 & 120 & C1-C2-C3-H8 & -180.0 \\
\hline $\mathrm{C} 5-\mathrm{C} 21$ & 1.005 & C3-C4-H9 & 120 & $\mathrm{Br} 25-\mathrm{C} 2-\mathrm{C} 3-\mathrm{C} 4$ & -180.0 \\
\hline C6-C10 & 1.000 & C5-C4-H9 & 120 & $\mathrm{Br} 25-\mathrm{C} 2-\mathrm{C} 3-\mathrm{H} 8$ & 0.0 \\
\hline C11-C12 & 1.359 & C4-C5-C6 & 120 & $\mathrm{C} 2-\mathrm{C} 3-\mathrm{C} 4-\mathrm{C} 5$ & 0.0 \\
\hline C11-S15 & 1.771 & C4-C5-C21 & 120 & C2-C3-C4-H9 & -179.9 \\
\hline C11-C19 & 1.005 & C6-C5-C21 & 120 & H8-C3-C4-C5 & 180.0 \\
\hline C12-C13 & 1.552 & C1-C6-C5 & 120 & H8-C3-C4-H9 & 0.0 \\
\hline C12-H16 & 1.000 & C1-C6-H10 & 120 & C3-C4-C5-C6 & 0.0 \\
\hline C13-C14 & 1.359 & C5-C6-H10 & 120 & C3-C4-C5-C21 & 179.9 \\
\hline C13-H17 & 1.000 & C12-C11-S15 & 110.5 & H9-C4-C5-C6 & 179.9 \\
\hline C14-S15 & 1.771 & C12-C11-C19 & 124.7 & H9-C4-C5-C21 & 0.0 \\
\hline C14-H18 & 1.000 & S15-C11-C19 & 124.7 & C4-C5-C6-C1 & 0.0 \\
\hline C19-C20 & 1.005 & C11-C12-C13 & 112.1 & C4-C5-C6-H10 & -180 \\
\hline $\mathrm{C} 19-\mathrm{O} 22$ & 1.258 & C11-C12-H16 & 123.9 & $\mathrm{C} 21-\mathrm{C} 5-\mathrm{C} 6-\mathrm{C} 1$ & -179.9 \\
\hline $\mathrm{O} 22-\mathrm{C} 21$ & 1.355 & C13-C12-H16 & 123.9 & C21-C5-C6-H10 & 0.0 \\
\hline $\mathrm{C} 20-\mathrm{H} 23$ & 1.000 & C12-C13-C13 & 112.1 & C4-C5-C21-C20 & -179.9 \\
\hline \multirow[t]{15}{*}{$\mathrm{C} 21-\mathrm{H} 24$} & 1.000 & C12-C13-H17 & 123.9 & C4-C5-C21-H24 & 0.0 \\
\hline & & C14-C13-H17 & 123.9 & C6-C5-C21-C20 & 0.0 \\
\hline & & C13-C14-S15 & 110.5 & C6-C5-C21-H24 & 179.9 \\
\hline & & C13-C14-H18 & 124.7 & $\mathrm{~S} 15-\mathrm{C} 11-\mathrm{C} 12-\mathrm{C} 13$ & -7.5 \\
\hline & & S15-C14-H18 & 124.7 & S15-C11-C12-H16 & 173.1 \\
\hline & & C11-S15-C14 & 93.3 & C19-C11-C12-C13 & 172.1 \\
\hline & & C11-C19-C20 & 120 & C19-C11-C12-H16 & -7.1 \\
\hline & & C11-C19-O22 & 120 & C12-C11-S15-C14 & 10.2 \\
\hline & & $\mathrm{C} 20-\mathrm{C} 19-\mathrm{O} 22$ & 120 & C19-C11-S15-C14 & -169.5 \\
\hline & & C19-C20-C21 & 120 & C12-C11-C19-C20 & 0.1 \\
\hline & & C19-C20-H23 & 120 & C12-C11-C19-O22 & -179.8 \\
\hline & & $\mathrm{C} 21-\mathrm{C} 20-\mathrm{H} 23$ & 120 & S15-C11-C19-C20 & 179.8 \\
\hline & & $\mathrm{C} 5-\mathrm{C} 21-\mathrm{C} 20$ & 120 & S15-C11-C19-O22 & -0.1 \\
\hline & & C5-C21-H24 & 120 & $\mathrm{C} 11-\mathrm{C} 12-\mathrm{C} 13-\mathrm{C} 14$ & 0.0 \\
\hline & & $\mathrm{C} 20-\mathrm{C} 21-\mathrm{H} 24$ & 120 & C11-C12-C13-H17 & -179.2 \\
\hline
\end{tabular}


RASĀYAN J. Chem.

Vol. 12 | No. 4 |2149 - 2165| October - December | 2019

\begin{tabular}{r|r|l|l|r|r}
\hline & & & & H16-C12-C13-C14 & 179.2 \\
\hline & & & & H16-C12-C13-H17 & 0.0 \\
\hline & & & & C12-C13-C14-S15 & 7.5 \\
\hline & & & & C12-C13-C14-H18 & -172.1 \\
\hline & & & & H17-C13-C14-S15 & -173.1 \\
\hline & & & & H17-C13-C14-H18 & 7.1 \\
\hline & & & & C13-C14-S15-C11 & -10.2 \\
\hline & & & & H18-C14-S15-C11 & 169.4 \\
\hline & & & & C11-C19-C20-C21 & 0.0 \\
\hline & & & & C11-C19-C20-H23 & -179.9 \\
\hline & & & & O22-C19-C20-C21 & -179.9 \\
\hline & & & & O22-C19-C20-H23 & 0.0 \\
\hline & & & & C19-C20-C21-C5 & 179.9 \\
\hline & & & & C19-C20-C21-H24 & 0.0 \\
\hline & & & & H23-C20-C21-C5 & 0.0 \\
\hline & & & & H23-C20-C21-H24 & 179.9 \\
\hline & & & & & \\
\hline
\end{tabular}

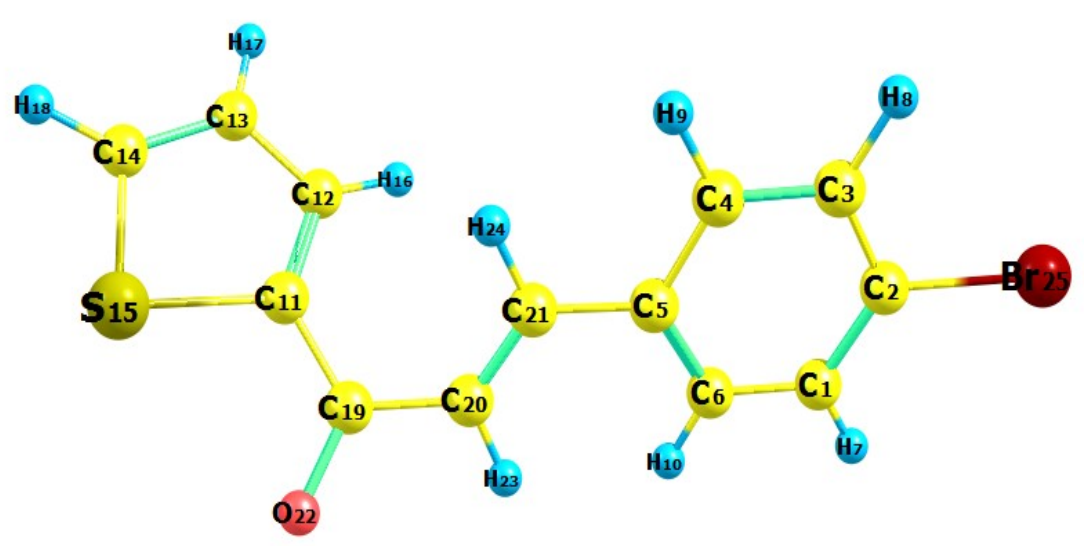

Fig.-1: Optimized Structure of 3-(4-Bromophenyl)-1-(thiophen-2-yl) prop-2-en-1 one.

\section{Vibrational Analysis}

The BPTP compound C1 symmetry system consists of 25 atoms and 69 normal vibrational modes. Vibration systems provide a systematic description of the FT-IR Fig.-2 and FT-Raman Fig. -2a and the TED analysis has been provided with basic criteria are shown in Table-2.

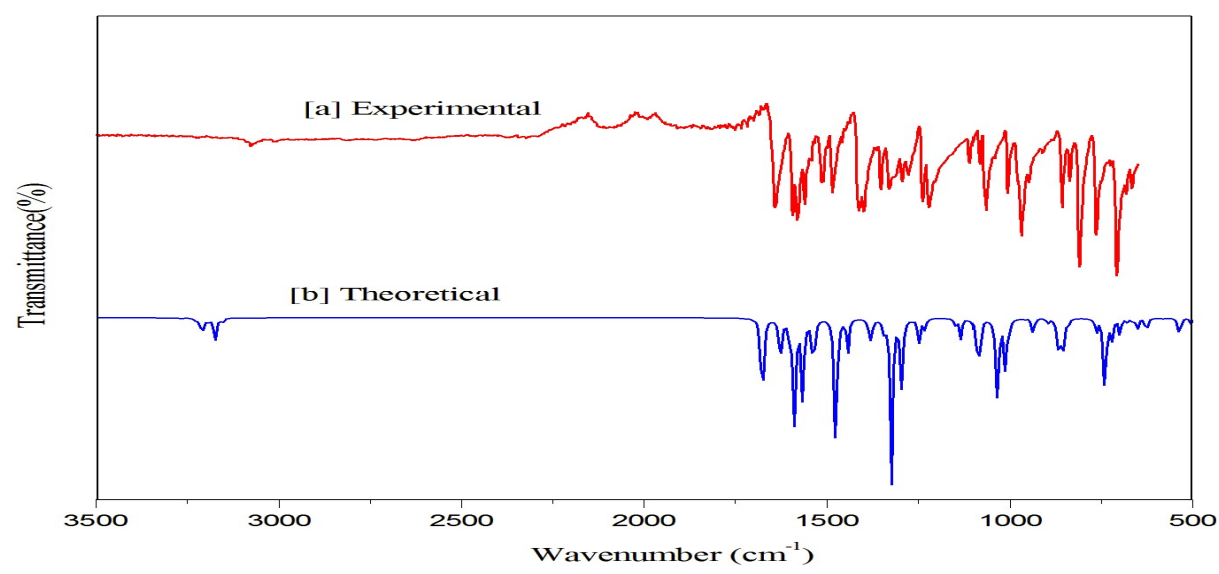

Fig.-2:[a]Experimental and [b]Theoretical FT-IR Spectrum of 3-(4-bromophenyl)-1-(thiophen-2-yl) prop-2-en-1 one 2152 
RASĀYAN J. Chem.

Vol. 12 | No. 4 |2149 - 2165| October - December | 2019

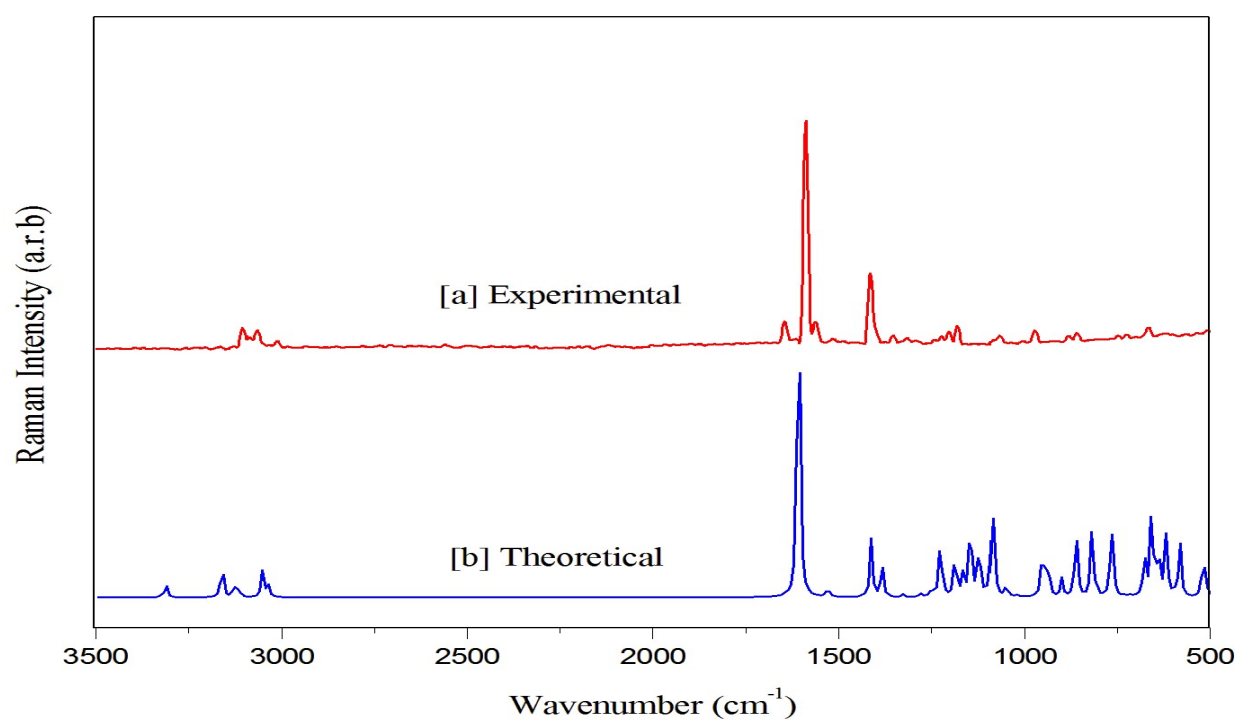

Fig.-2a: [a] Experimental and [b] Theoretical FT-Raman Spectrum of 3-(4-bromophenyl)-1-(thiophen-2-yl) prop-2en-1 one.

Table -2: Vibrational Wave Numbers Obtained BPTP at 6-311G (d,p) Method [ Harmonic Frequency $\left(\mathrm{cm}^{-1}\right), I_{R}$ int $\left(\mathrm{kmmol}^{-1}\right)$ ] Raman Intensity (Arb Units)].

\begin{tabular}{|c|c|c|c|c|c|c|}
\hline \multirow[t]{2}{*}{$\begin{array}{l}\text { Mode } \\
\text { nos. }\end{array}$} & \multicolumn{2}{|c|}{$\begin{array}{c}\text { Experimental } \\
\text { Wavenumber }\left(\mathrm{cm}^{-1}\right)\end{array}$} & \multicolumn{3}{|c|}{$\begin{array}{c}\text { Theoretical } \\
\text { Wavenumber }\left(\mathrm{cm}^{-1}\right)\end{array}$} & \multirow[t]{2}{*}{$\begin{array}{l}\text { TED }(\geq 10 \%) \\
\text { Assignments }\end{array}$} \\
\hline & FT-IR & FT-Raman & $\begin{array}{c}\text { B3LYP } \\
\text { scaled }\end{array}$ & $\mathrm{IR}_{\text {int }}$ & $\operatorname{Ram}_{\text {Int }}$ & \\
\hline 1 & $3154 \mathrm{vw}$ & & 3158 & 5.30 & 50.09 & $\gamma \mathrm{CH}(95)$ \\
\hline 2 & & & 3118 & 21.88 & 159.21 & $\gamma \mathrm{CH}(97)$ \\
\hline 3 & & & 3107 & 0.25 & 368.85 & $\gamma \mathrm{CH}(96)$ \\
\hline 4 & & $3105 \mathrm{w}$ & 3105 & 3.30 & 111.48 & $\gamma \mathrm{CH}(80)$ \\
\hline 5 & & & 3099 & 8.44 & 171.89 & $\gamma \mathrm{CH}(97)$ \\
\hline 6 & $3074 \mathrm{vw}$ & & 3085 & 8.30 & 269.53 & $\gamma \mathrm{CH}(97)$ \\
\hline 7 & & $3067 \mathrm{w}$ & 3068 & 0.89 & 53.91 & $\gamma \mathrm{CH}(91)$ \\
\hline 8 & & & 3066 & 1.12 & 67.49 & $\gamma \mathrm{CH}(93)$ \\
\hline 9 & $3009 w$ & $3012 \mathrm{vw}$ & 3049 & 164.32 & 54.62 & $\gamma \mathrm{CH}(94)$ \\
\hline 10 & $1638 \mathrm{vs}$ & $1645 w$ & 1621 & 34.22 & 35.91 & $\gamma \mathrm{CC}(57)+\beta \mathrm{HCC}(23)$ \\
\hline 11 & $1582 \mathrm{vs}$ & $1589 \mathrm{vs}$ & 1573 & 153.04 & 233.80 & $\gamma \mathrm{CH}(30)$ \\
\hline 12 & & $1562 \mathrm{~m}$ & 1547 & 12.71 & 7.03 & $\gamma \mathrm{CC}(41)+\beta \mathrm{CCC}(11)$ \\
\hline 13 & & & 1536 & 19.41 & 5.79 & $\gamma \mathrm{OC}(41)+\gamma \mathrm{CCC}(19)$ \\
\hline 14 & $1511 \mathrm{~m}$ & $1515 \mathrm{vw}$ & 1514 & 39.07 & 514.29 & $\gamma \mathrm{OC}(43)+\gamma \mathrm{CC}(33)+\beta \mathrm{HCC}(10)$ \\
\hline 15 & $1481 \mathrm{~m}$ & & 1486 & 9.87 & 16.65 & $\beta \mathrm{HCC}(68)$ \\
\hline 16 & $1404 \mathrm{vs}$ & $1414 s$ & 1427 & 107.48 & 526.31 & $\gamma \mathrm{CC}(42)+\beta \mathrm{HCC}(13)$ \\
\hline 17 & & & 1395 & 32.22 & 64.16 & $\beta \mathrm{HCC}(28)+, \gamma \mathrm{CC}(26)$ \\
\hline 18 & $1350 \mathrm{~m}$ & $1352 \mathrm{vw}$ & 1341 & 15.05 & 63.15 & $\beta \mathrm{HCC}(54)+\gamma \mathrm{CC}(10)$ \\
\hline 19 & $1322 \mathrm{~m}$ & & 1332 & 24.89 & 7.24 & $\beta \mathrm{HCS}(25)+\beta \mathrm{HCC}(21)+\gamma \mathrm{CC}(12)$ \\
\hline 20 & & $1314 \mathrm{vm}$ & 1310 & 84.33 & 103.96 & $\beta \mathrm{HCC}(70)$ \\
\hline 21 & $1291 w$ & & 1298 & 17.56 & 8.84 & $\beta \mathrm{HCC}(48)+\gamma \mathrm{CC}(10)$ \\
\hline 22 & & & 1279 & 28.66 & 4.11 & $\gamma \mathrm{CC}(30)$ \\
\hline
\end{tabular}


RASĀYAN J. Chem.

Vol. 12 | No. 4 |2149 - 2165| October - December | 2019

\begin{tabular}{|c|c|c|c|c|c|c|}
\hline 23 & & & 1252 & 100.33 & 25.67 & $\gamma \mathrm{CC}(16)+\beta \mathrm{HCC}(12)$ \\
\hline 24 & & & 1225 & 15.37 & 59.72 & $\beta \mathrm{HCC}(38), \gamma \mathrm{CC}(14)$ \\
\hline 25 & 1216vs & $1202 \mathrm{vw}$ & 1206 & 41.23 & 30.04 & $\gamma \mathrm{CC}(44)+\beta \mathrm{HCC}(11)$ \\
\hline 26 & & $1180 \mathrm{w}$ & 1191 & 41.23 & 363.81 & $\beta \mathrm{HCC}(68)$ \\
\hline 27 & $1109 \mathrm{w}$ & & 1111 & 57.38 & 15.40 & $\beta \mathrm{HCC}(41)+\gamma \mathrm{CC}(27)$ \\
\hline 28 & & & 1096 & 63.80 & 32.54 & $\beta \mathrm{HCS}(38)+\beta \mathrm{HCC}(33)+\gamma \mathrm{CC}(13)$ \\
\hline 29 & 1063vs & $1067 \mathrm{vw}$ & 1053 & 3.67 & 0.70 & $\gamma \mathrm{CC}(53)$ \\
\hline 30 & & & 1047 & 1.99 & 18.11 & $\gamma \mathrm{CC}(47)+\beta \mathrm{HCC}(15)+\beta \mathrm{HCS}(14)$ \\
\hline 31 & $1003 \mathrm{w}$ & & 1003 & 37.56 & 14.25 & $\beta C C C(81)$ \\
\hline 32 & & & 1000 & 1.46 & 2.98 & $\tau \mathrm{HCCC}(70)$ \\
\hline 33 & & & 980 & 34.34 & 7.76 & $\beta \mathrm{HCC}(22)+\gamma \mathrm{CC}(14)$ \\
\hline 34 & & $973 w$ & 978 & 15.94 & 37.07 & $\tau \mathrm{HCCC}(41)$ \\
\hline 35 & & & 968 & 5.87 & 169.38 & $\tau \mathrm{CCCC}(38)+\tau \mathrm{HCCC}(31)$ \\
\hline 36 & & & 930 & 15.40 & 8.71 & $\tau \mathrm{HCCS}(43)+\tau \mathrm{HCCC}(29)+\mathrm{CCCC}(13)$ \\
\hline 37 & & $882 \mathrm{vw}$ & 906 & 17.37 & 5.17 & $\tau \mathrm{HCCC}(72)$ \\
\hline 38 & $854 \mathrm{~m}$ & $867 \mathrm{w}$ & 867 & 55.46 & 156.47 & $\tau \mathrm{HCCC}(72)+\tau \mathrm{HCCS}(20)+\tau \mathrm{HCSC}(19)$ \\
\hline 39 & & $867 \mathrm{w}$ & 840 & 28.23 & 27.79 & $\beta C C C(19)$ \\
\hline 40 & & & 835 & 10.35 & 14.04 & $\tau \mathrm{HCCC}(98)$ \\
\hline 41 & & & 829 & 68.06 & 5.24 & $\tau \mathrm{HCCC}(45)$ \\
\hline 42 & $807 \mathrm{vs}$ & & 815 & 6.05 & 23.09 & $\beta C C C(53)$ \\
\hline 43 & $750 \mathrm{~s}$ & & 739 & 58.81 & 59.03 & $\beta \mathrm{HCCC}(25)+\tau \mathrm{OCCC}(18)+\tau \mathrm{CCCC}(12)$ \\
\hline 44 & & & 716 & 4.75 & 38.02 & $\tau \mathrm{HCCSC}(65)+\tau \mathrm{HCCS}(25)$ \\
\hline 45 & & & 698 & 25.96 & 52.38 & $\tau \mathrm{CCCC}(10)+\delta \mathrm{OCCC}(20)$ \\
\hline 46 & & $667 \mathrm{w}$ & 676 & 21.40 & 66.20 & $\gamma \mathrm{SC}(29)+\beta \mathrm{SCC}(17)+\delta \mathrm{OCCC}(12)$ \\
\hline 47 & & $653 \mathrm{vw}$ & 656 & 19.56 & 78.76 & $\gamma \mathrm{SC}(30)$ \\
\hline 48 & & & 641 & 40.89 & 5.73 & $\beta C C C(51)$ \\
\hline 49 & & & 630 & 51.48 & 13.19 & $\beta \mathrm{SCC}(28)+\beta \mathrm{CCC}(23)$ \\
\hline 50 & & & 605 & 23.56 & 29.82 & $\beta \mathrm{OCC}(31)+\gamma \mathrm{SC}(18)$ \\
\hline 51 & & & 562 & 30.23 & 11.63 & $\tau \mathrm{CCCC}(66)+\tau \mathrm{HCCC}(11)$ \\
\hline 52 & & & 519 & 38.37 & 19.35 & $\tau \mathrm{CCCC}(18)+\beta \mathrm{SCC}(10)$ \\
\hline 53 & & & 486 & 10.08 & 298.58 & $\beta \mathrm{SCC}(18)+\beta \mathrm{CCC}(12)$ \\
\hline 54 & & & 438 & 10.48 & 20.47 & $\tau \operatorname{SCCC}(10)$ \\
\hline 55 & & & 414 & 0.00 & 245.46 & $\tau \mathrm{CCCC}(58)+\tau \mathrm{HCCC}(10)$ \\
\hline 56 & & & 399 & 0.57 & 7.29 & $\beta \mathrm{CCC}(26)+\gamma \mathrm{CC}(10)$ \\
\hline 57 & & & 373 & 11.43 & 49.53 & $\beta \mathrm{HCC}(54)+\gamma \mathrm{CC}(20)+\gamma \mathrm{BrCC}(12)$ \\
\hline 58 & & & 337 & 1.84 & 85.06 & $\delta \mathrm{BrCCC}(22)+\delta \mathrm{CCCC}(15)$ \\
\hline 59 & & & 282 & 0.45 & 5.61 & $\gamma \mathrm{BrC}(29)+\gamma \mathrm{BrCC}(12)$ \\
\hline 60 & & & 224 & 10.30 & 81.90 & $\beta \mathrm{BrCC}(56)$ \\
\hline 61 & & $183 \mathrm{vw}$ & 196 & 0.23 & 30.60 & $\beta \mathrm{CCC}(26)+\tau \mathrm{CCCC}(14)+\delta \mathrm{BrCCC}(14)$ \\
\hline 62 & & & 174 & 1.27 & 4.28 & $\tau \mathrm{CCCC}(23)+\beta \mathrm{CCC}(18)$ \\
\hline 63 & & & 159 & 0.38 & 8.60 & $\tau \mathrm{CCCC}(65)$ \\
\hline 64 & & & 136 & 2.11 & 4.00 & $\tau \mathrm{CCCC}(45)+\delta \mathrm{OCCC}(11)$ \\
\hline 65 & & $89 \mathrm{vw}$ & 103 & 0.66 & 8.67 & $\beta \mathrm{CCC}(41)+\tau \mathrm{CCCC}(20)$ \\
\hline 66 & & & 76 & 2.65 & 10.22 & $\beta \mathrm{CCC}(10)+\tau \mathrm{CCCC}(40)$ \\
\hline 67 & & & 36 & 172.68 & 39.40 & $\gamma \mathrm{CC}(15)+\beta \mathrm{CCC}(13)$ \\
\hline 68 & & & 31 & 178.20 & 1485.23 & $\tau \mathrm{CCCC}(58)+\beta \operatorname{BrCC}(56)+\delta \mathrm{CCCC}(13)$ \\
\hline 69 & & & 24 & 524.63 & 404.18 & $\tau \mathrm{CCCC}(55)+\delta \mathrm{OCCC}(17)$ \\
\hline
\end{tabular}

Ram $_{\text {Int }}$ - Raman Intensity; IR int $^{-}$IR intensity; Kmmol ${ }^{-1}$ s-strong; vs-very strong; m-medium; w-weak; vw- very weak $\gamma$ - stretching; $\delta$ - out-of -plane bending; $\beta$ - in plane bending; $\tau$ - torsion. 
RASĀYAN J. Chem.

Vol. 12 | No. 4 |2149 - 2165| October - December | 2019

\section{C-H Vibration}

The BPTP compound $9 \mathrm{CH}$ stretching vibration and the $\mathrm{C}-\mathrm{H}$ vibration commonly exhibits weak region at $3100-3000 \mathrm{~cm}^{-1} 7,8$ and they are not generally affected appreciably by the common substitutions. C-H stretching vibration normally pure mode contribution observed at $3049-3158 \mathrm{~cm}^{-1}$.FT-IR bands observed at 3074(w)-3074(w) and FT-Raman observed peaks at 3105(w), 3067(w) and 3012(vw) are a good agreement with each other.

The HCC stretching vibrational in-plane bending region $1627-1087 \mathrm{~cm}^{-1}$. FT-IR bands observed at 1638(vs), 1511(m), 1481(m), 1404(vs), 1350(m), 1322(m), 1291(w), 1216(vs) and 1109(w) and Raman bands observed at $1645(\mathrm{w}), 1562(\mathrm{~m}), 1515(\mathrm{vw}), 1414(\mathrm{~s}), 1352(\mathrm{vw}), 1314(\mathrm{vw}), 1202(\mathrm{vw})$ and $1180(\mathrm{w})$ commonly. Torsion vibration of HCCC assessment at $1034-412 \mathrm{~cm}^{-1}$. FT-IR observed bands at 854(m) and in FT-Raman observed peaks at 973(w),882(vw) and 867(w) and the harmonic frequency with TED value $97 \%$.

\section{$\mathbf{C}=\mathbf{O}$ Vibration}

The $\mathrm{C}=\mathrm{O}$ vibration pi-bonding between oxygen and carbon atoms. The $\mathrm{C}=\mathrm{O}$ stretching vibration is observed in the region $1680-1715 \mathrm{~cm}^{-1} .{ }^{9}$ This case calculated $\mathrm{C}=\mathrm{O}$ stretching vibration present in the region 1514-1536 $\mathrm{cm}^{-1}$. FT-IR bands observed at $1515(\mathrm{vw})$ and FT-Raman observed bands at $1511(\mathrm{~m})$ and 1561(w). TED contribution at $43 \%$. $\mathbf{C}=\mathbf{C}$ and $\mathbf{C}-\mathbf{C}$ vibrations.

The $\gamma(C-C)$ stretching vibrations are expected bands range of $1590-1640,1560-1580$ and $1470-1510 \mathrm{~cm}^{-1}{ }^{10}$ The theoretical wavenumber for $\gamma(\mathrm{C}-\mathrm{C})$ mode lies at $36-1621 \mathrm{~cm}^{-1}$ with TED value $57 \%$. In this present study FT-IR bands observed 1638(vs), 1582(vs), 1404(vs),1350(m), 1322(m) and 1291(w) and in FTRaman observed at the bands 1645(w), 1589(vs),1562(m), 1515(vw),1414(s), 1352(vw), 1202(vw) and $1167(\mathrm{vw})$ are designated as $\gamma \mathrm{C}-\mathrm{C}$ vibrations. The $\beta C C \mathrm{C}$ in-plane bending region observed at $1583-37 \mathrm{~cm}^{-}$ ${ }^{1}$ at TED value $11 \%$. FT-IR observed bands at $1003 \mathrm{~cm}^{-1}$ (weak) and in Raman peaks observed at $1562(\mathrm{~m})$ and $89(\mathrm{vw})$.

\section{C-Br Vibration}

The BPTP title compound C- Br, vibrational in the region $650-395 \mathrm{~cm}^{-1} .{ }^{11}$ This absorbs the stretching vibration. The stretching vibrations present in the part of 373-282 $\mathrm{cm}^{-1}$. In-plane bending observed mode $224-31 \mathrm{~cm}^{-1}$ and out of bending mode $337-196 \mathrm{~cm}^{-1}$. The computational vibrational frequency appears in 373-196 $\mathrm{cm}^{-1}$.FT-Raman bands at 183(vw) with TED 56\%.

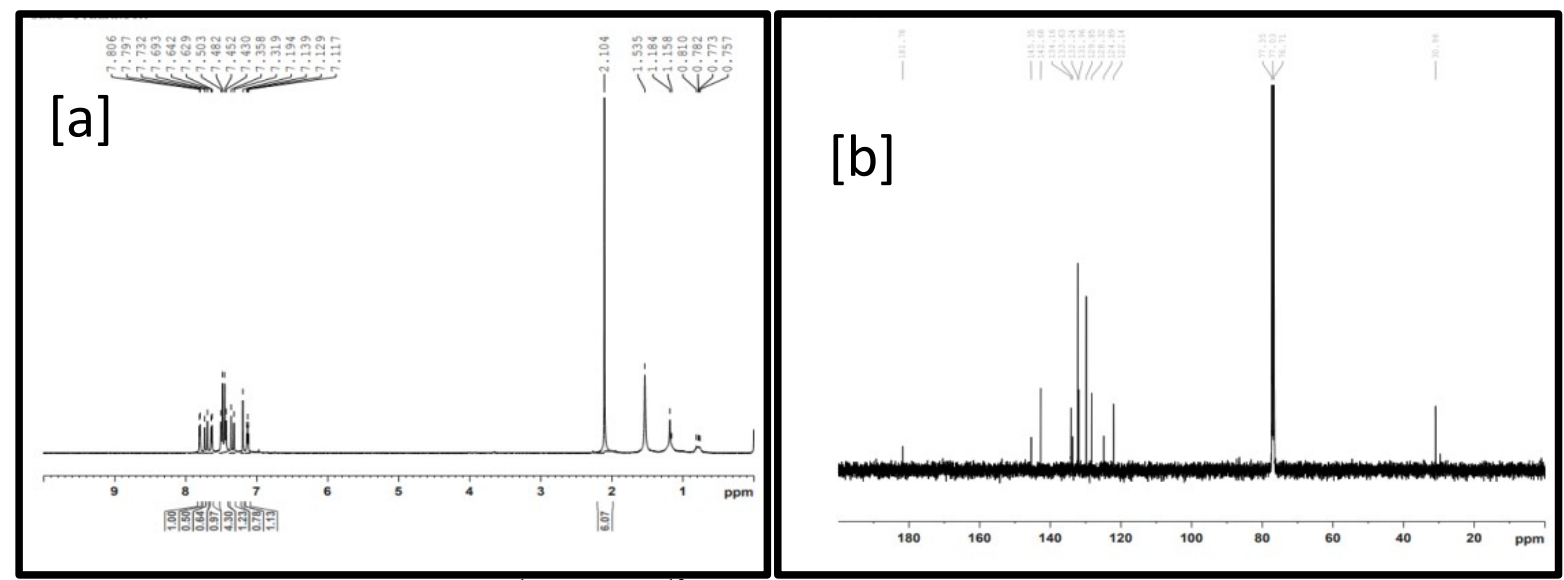

Fig.-4: NMR Spectrum of [a] ${ }^{1} \mathrm{H}$ and $[\mathrm{b}]{ }^{13} \mathrm{C}$ 3-(4-bromophenyl)-1-(thiophene-2-yl) prop-2-en-1 one

\section{Thiophene Ring Vibration}

In common, the assignment of vibration in the alternate compound is arduous in the infrared. The adsorption of this group is of unstable intensity and may be found over a broad part $1035-245 \mathrm{~cm}^{-1}$ both aromatic and aliphatic sulphide. ${ }^{12}$ FT-Raman bands at $667(\mathrm{w})$ and $653(\mathrm{vw})$ and the C-S stretching mode TED contribution $30 \%$. 
RASĀYAN J. Chem.

Vol. 12 | No. 4 |2149 - 2165| October - December | 2019

\section{NMR Spectral Analysis}

The proton and carbon nuclear magnetic resonance spectra were calculated to confirm the target compound structure. Both ${ }^{1} \mathrm{H}$ and ${ }^{13} \mathrm{CNMR}$ spectra (Experimental and theoretical) have seen in Fig-3. The proton NMR, a multiplet peak presented in the constituent 7.11-7.80 ppm with 9 proton entire value barely arrangement to the aromatic ring protons. The ${ }^{1} \mathrm{H}$ signals were theoretically calculated and it is received between the constituent 6.75-8.57ppmvalues are good agreement shown in Table-3.The carbon signals at 122.14-145.35 are due to the presence in the aromatic ring carbons. The signal at $181.75 \mathrm{ppm}$ is due to the $\mathrm{C}=\mathrm{O}$ carbon present in the compound. All the theoretical values are a good agreement with the experimental one.

Table -3: The Predicted and Observed ${ }^{1} \mathrm{H}$ and ${ }^{13} \mathrm{C}$ Isotopic Chemical Shifts for BPTP

\begin{tabular}{|c|c|c|c|}
\hline \multirow[b]{2}{*}{ Atom Number } & \multicolumn{2}{|c|}{ B3LYP/6-311G(d.p.) } & \multirow[b]{2}{*}{ Experimenta } \\
\hline & Gas Phase & Solvent Phase & \\
\hline $2 \mathrm{C}$ & 178.62 & 178.88 & 176.34 \\
\hline $3 \mathrm{C}$ & 162.71 & 163.97 & - \\
\hline $4 \mathrm{C}$ & 147.15 & 149.09 & - \\
\hline $5 \mathrm{C}$ & 145.88 & 145.51 & 145.35 \\
\hline $6 \mathrm{C}$ & 140.05 & 141.27 & 142.58 \\
\hline $8 \mathrm{C}$ & 139.64 & 140.24 & 134.16 \\
\hline $10 \mathrm{C}$ & 139.43 & 139.37 & 133.63 \\
\hline $12 \mathrm{C}$ & 137.29 & 138.95 & 132.24 \\
\hline $13 \mathrm{C}$ & 136.78 & 136.13 & 131.96 \\
\hline $14 \mathrm{C}$ & 134.34 & 134.27 & 129.85 \\
\hline $15 \mathrm{C}$ & 126.23 & 127.27 & 128.32 \\
\hline $16 \mathrm{C}$ & 125.30 & 126.15 & 124.89 \\
\hline $17 \mathrm{C}$ & 118.52 & 119.64 & 122.14 \\
\hline $11 \mathrm{H}$ & 8.37 & 8.57 & 7.80 \\
\hline $9 \mathrm{H}$ & 7.69 & 7.99 & 7.75 \\
\hline $19 \mathrm{H}$ & 7.65 & 7.87 & 7.71 \\
\hline $20 \mathrm{H}$ & 7.71 & 7.64 & 7.52 \\
\hline $21 \mathrm{H}$ & 7.58 & 7.70 & 7.50 \\
\hline $22 \mathrm{H}$ & 7.26 & 7.48 & 7.32 \\
\hline $23 \mathrm{H}$ & 6.92 & 7.14 & 7.31 \\
\hline $24 \mathrm{H}$ & 6.90 & 7.13 & 7.19 \\
\hline $25 \mathrm{H}$ & 6.53 & 6.75 & 7.13 \\
\hline
\end{tabular}

\section{UV-VIS Spectral Analysis}

The TD-DFT calculation has been carried out using B3LYP/6-311G (d,p) basis set and concluded the low-lying excited states of BPTP were present. In this calculation both solvent phase and gas and employing ethanol environment. Obtained computed values are given in Table-4 and the spectrum was shown in Fig. $-4 .{ }^{13}$ The theoretical data at $496.97,319.27$ and $305.60 \mathrm{~nm}$ in the gas phase, 494.95318 .65 and $304.70 \mathrm{~nm}$. The measured oscillator strength of BPTP found to be $0.0039(496.96), 02180(319.27 \mathrm{~nm})$ and $0.0079(305.60) \mathrm{nm}$ in the gas phase, and $0.0039(494.95), 0.220(318.65 \mathrm{~nm})$ and $0.0078(304.70 \mathrm{~nm})$ in ethanol medium.

The predicted $\pi \rightarrow \pi^{*}$ transitions was responsible for HOMO $\rightarrow$ LUMO $(95 \%), \mathrm{H}-12 \rightarrow$ LUMO $(3 \%), \mathrm{H}-$ $1 \rightarrow$ LUMO $(82 \%), \mathrm{H}-4 \rightarrow$ LUMO $(7 \%), \mathrm{H}-3 \rightarrow$ LUMO $(5 \%)$ and $\mathrm{H}-4 \rightarrow$ LUMO $(14 \%), \mathrm{H}-2 \rightarrow$ LUMO (77\%), H-3 $\rightarrow$ LUMO (2\%), H-1 $\rightarrow$ LUMO (3\%) ethanol solvent also transition under Gas-phase mode, It is there HOMO $\rightarrow$ LUMO $(95 \%), \mathrm{H}-12 \rightarrow$ LUMO $(3 \%), \mathrm{H}-1 \rightarrow$ LUMO $(83 \%), \mathrm{H}-4 \rightarrow$ LUMO $(7 \%), \mathrm{H}-$ $\rightarrow$ LUMO $5 \%$ ) and H-4 $\rightarrow$ LUMO (14\%), H-2 $\rightarrow$ LUMO (77\%), H-1 $\rightarrow$ LUMO (3\%). ${ }^{13}$ 
RASĀYAN J. Chem.

Vol. 12 | No. 4 |2149 - 2165| October - December | 2019

\section{Fukui Function}

The Fukui functions in between a simpler and normal-enabled reaction indicator the replacement of the Fukui function of the molecular density function $\rho \mathrm{N}(\mathrm{r})$. The Fukui function indices are the calculation of chemical reactivity, an indicator of reactive place and the electrophilic and nucleophilic reaction of the molecules it is to identify the electron acceptor center and donor centers. If $\mathrm{f}+$ is real value then the imaginary value of $\mathrm{f}$ - implies electrophilic attract.

The Fukui indices for summation of electron to a BPTP are given by:

$\mathrm{f}+(\mathrm{r})=\rho \mathrm{N}+1(\mathrm{r})-\rho \mathrm{N}(\mathrm{r})$
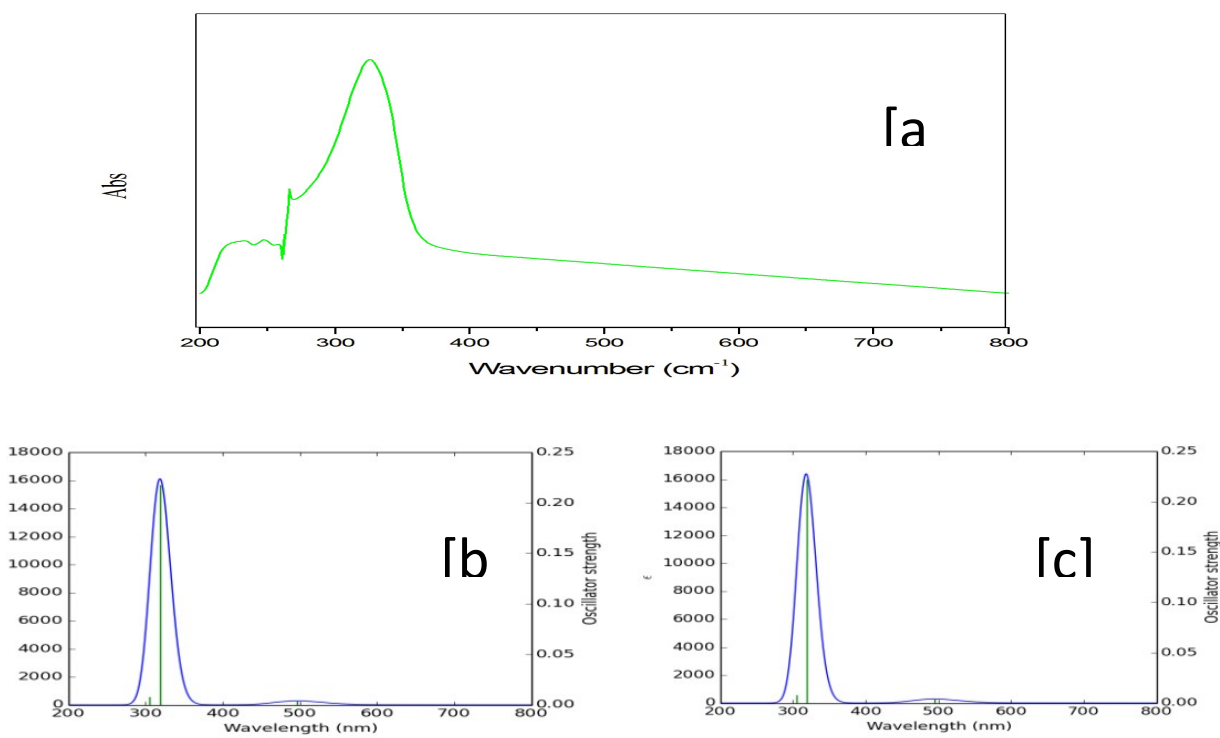

Fig.-4: Optical Absorption Spectrum of 3-(4-Bromophenyl)-1-(thiophen-2-yl) prop-2-en-1 one [a] Experimental, [b]Simulated in Ethanol and [c] Gas Phase Using TD-DFT method.

Table-4: Experimental and Calculated Absorption Wavelength, Energies and Oscillator Strengths of 3-(4bromophenyl)-1-(thiophene-2-yl) prop-2-en-1 one using the TD-DFT Method of B3LYP/6-311G (d,p) Method.

\begin{tabular}{|c|c|c|c|c|c|c|c|}
\hline & Excitation & $\begin{array}{c}\text { CI } \\
\text { Expansion } \\
\text { Coefficient }\end{array}$ & $\begin{array}{c}\text { Wavelength } \\
\lambda \\
(\mathrm{nm}) \\
\end{array}$ & $\begin{array}{l}\text { Oscillator } \\
\text { Strength } \\
\text { (f) }\end{array}$ & Expt. & Assign. & $\begin{array}{l}\text { In Solvent } \\
{ }^{\text {a }} \text { Major contribution } \\
(\geq 10 \%)\end{array}$ \\
\hline \multirow{4}{*}{$\begin{array}{l}\text { Cal. } \\
\text { DMSO } \\
\text { phase }\end{array}$} & $\begin{array}{l}\text { Excited } \\
\text { State } 1 \\
\end{array}$ & & & & & & \\
\hline & $\begin{array}{l}69->74 \\
73->74 \\
\end{array}$ & $\begin{array}{c}-0.12995 \\
0.68878 \\
\end{array}$ & 496.97 & 0.0039 & & $\begin{array}{l}\pi \rightarrow \pi^{*} \\
\pi \rightarrow \pi^{*}\end{array}$ & $\begin{array}{l}\text { HOMO } \rightarrow \text { LUMO } \\
(95 \%)\end{array}$ \\
\hline & $\begin{array}{c}\text { Excitated } \\
\text { State } 2 \\
69->74 \\
70->74 \\
72->74 \\
\end{array}$ & $\begin{array}{l}0.19164 \\
0.16069 \\
0.64185 \\
\end{array}$ & 319.27 & 0.2180 & 325 & $\begin{array}{l}\pi \rightarrow \pi^{*} \\
\pi \rightarrow \pi^{*} \\
\pi \rightarrow \pi^{*}\end{array}$ & H-1 $\rightarrow$ LUMO (82\%) \\
\hline & $\begin{array}{c}\text { Excitated } \\
\text { State } 3 \\
69->74 \\
70->74 \\
71->74 \\
72->74 \\
\end{array}$ & $\begin{array}{c}-0.26435 \\
-0.10078 \\
0.61979 \\
0.12112 \\
\end{array}$ & 305.60 & 0.0079 & & $\begin{array}{l}\pi \rightarrow \pi^{*} \\
\pi \rightarrow \pi^{*} \\
\pi \rightarrow \pi^{*}\end{array}$ & $\mathrm{H}-2 \rightarrow \mathrm{LUMO}(77 \%)$ \\
\hline \multirow{2}{*}{$\begin{array}{l}\text { Cal. } \\
\text { Gas- }\end{array}$} & $\begin{array}{l}\text { Excited } \\
\text { State } 1\end{array}$ & & & & & & \\
\hline & $69->74$ & -0.26832 & 304.70 & 0.0078 & & $\pi \rightarrow \pi^{*}$ & HOMO $\rightarrow$ LUMO $(95 \%)$ \\
\hline
\end{tabular}


RASĀYAN J. Chem.

Vol. 12 | No. 4 |2149 - 2165| October - December | 2019

\begin{tabular}{|c|c|c|c|c|c|c|}
\hline \multirow[t]{5}{*}{ phase } & $\begin{array}{l}71->74 \\
72->74\end{array}$ & $\begin{array}{l}0.61970 \\
0.11737\end{array}$ & & & $\pi \rightarrow \pi^{*}$ & \\
\hline & $\begin{array}{l}\text { Excited } \\
\text { State } 2\end{array}$ & & & & & \\
\hline & $\begin{array}{l}69->74 \\
73->74\end{array}$ & $\begin{array}{r}-0.13065 \\
0.68885\end{array}$ & 494.95 & 0.0039 & $\begin{array}{l}\pi \rightarrow \pi^{*} \\
\pi \rightarrow \pi^{*} \\
\pi \rightarrow \pi^{*}\end{array}$ & $\mathrm{H}-1 \rightarrow$ LUMO $(83 \%)$ \\
\hline & $\begin{array}{l}\text { Excited } \\
\text { State } 3\end{array}$ & & & & & \\
\hline & $\begin{array}{l}69->74 \\
70->74 \\
72->74\end{array}$ & $\begin{array}{l}0.19004 \\
0.15753 \\
0.64327\end{array}$ & 318.65 & 0.2220 & $\begin{array}{l}\pi \rightarrow \pi^{*} \\
\pi \rightarrow \pi^{*} \\
\pi \rightarrow \pi^{*}\end{array}$ & $\mathrm{H}-2 \rightarrow$ LUMO $(77 \%)$ \\
\hline
\end{tabular}

The Fukui indices for removal of an electron from a BPTP are given by:

$f-(r)=\rho N(r)-\rho N-1(r)$

The first part of an $\mathrm{f}+$ function represents a nucleophilic reaction. On the other hand, the first part of an $\mathrm{f}-$ function represents electrophilic reaction. The Fukui indices (f),global and local electrophilicity index $(\omega)$ and global-local softness (S) of the molecule under study are presented in Table-5. It is seen that the atoms $7 \mathrm{C}, 8 \mathrm{C}, 9 \mathrm{H}, 10 \mathrm{C}, 11 \mathrm{H}, 12 \mathrm{C}, 15 \mathrm{C}, 16 \mathrm{C}, 18 \mathrm{Br}, 19 \mathrm{H}, 23 \mathrm{H}$, and $24 \mathrm{H}$ are good nucleophiles having negative local electrophilic index $\Delta \omega$ and they are ready to donate an electron pair to form a bond and which is capable to electrophilic attack. The atoms $1 \mathrm{C}, 2 \mathrm{C}, 3 \mathrm{C}, 4 \mathrm{C}, 6 \mathrm{C}, 13 \mathrm{C}, 14 \mathrm{C}, 17 \mathrm{C}, 20 \mathrm{H}, 22 \mathrm{H}$, and $25 \mathrm{H}$ are good electrophiles having positive local electrophilic index $\Delta \omega$ and they are ready to accept electron pair to form a bond and which is capable to nucleophilic attack. ${ }^{14}$

Table-5: Fukui Function and Global and Local Softness, and Electrophlicity Index of Fig.-1 Optimized Structure of 3-(4-Bromophenyl)-1-(thiophen-2-yl) prop-2-en-1 one.

\begin{tabular}{c|c|c|c|c}
\hline $\mathrm{f}+=(\mathrm{q}+1)-\mathrm{q}$ & $\mathrm{f}-=\mathrm{q}-(\mathrm{q}+1)$ & $\Delta \mathrm{f}=(\mathrm{f}+)-(\mathrm{f}-)$ & $\Delta \mathrm{f}=\Delta \mathrm{f} \mathrm{S}_{\mathrm{gs}}$ & $\Delta \omega=\Delta \mathrm{f} \omega_{\mathrm{ei}}$ \\
\hline-0.12867 & 0.53157 & -0.66024 & 0.17261 & -0.90545 \\
\hline-3.68903 & 0.06673 & -3.75576 & 0.98194 & -5.15060 \\
\hline-0.13039 & 0.10698 & -0.23737 & 0.06206 & -0.32553 \\
\hline-0.13912 & 0.07310 & -0.21222 & 0.05548 & -0.29104 \\
\hline-0.07270 & -0.16882 & 0.09612 & -0.02513 & 0.13182 \\
\hline-0.04728 & 0.36938 & -0.41667 & 0.10893 & -0.57142 \\
\hline-0.47124 & 0.66172 & -1.13296 & 0.03476 & -1.55374 \\
\hline 0.52241 & -0.70769 & -0.18528 & 0.04844 & -0.25409 \\
\hline-0.10245 & 0.13498 & -0.23743 & 0.06207 & -0.32562 \\
\hline-0.43625 & 0.00000 & -0.43625 & 0.11405 & -0.64031 \\
\hline-0.38942 & -0.44039 & -0.82981 & -0.21695 & 1.13800 \\
\hline 0.30243 & 0.16446 & 0.46690 & 0.04585 & 0.64031 \\
\hline-0.18787 & 0.33202 & -0.51989 & 0.13592 & -0.71298 \\
\hline-0.08408 & 0.09130 & -0.17538 & -0.12207 & -0.24052 \\
\hline-0.36476 & 0.50124 & -0.86600 & 0.22641 & -0.18764 \\
\hline-0.411302 & -0.07547 & -0.33582 & 0.88780 & -0.46055 \\
\hline
\end{tabular}

\section{Dipole Moment}

The target compound obtained dipole moment is 4.2457. The three axes represent the three directions and these are XYZ Cartesian coordinates. X-axis 4-bromophenyl direction, however, refers to the $\mathrm{Y}$-axis chalcone moieties direction Dipole moment match with the water the dipole moment value is higher than the water value. This BPTP is a white color compound and has medicinal properties is shown in Fig-5 and Table- $6 .^{16}$ 
RASĀYAN J. Chem.

Vol. 12 | No. 4 |2149 - 2165| October - December | 2019

Table -6: Dipole Moment Components of 3-(4-Bromophenyl)-1-(thiophen-2-yl) prop-2-en-1 one

\begin{tabular}{c|c}
\hline Dipole vector components & Dipole moment, $\mu($ Debye $)$ \\
\hline$\mu_{\mathrm{x}}$ & 1.0174 \\
\hline$\mu_{\mathrm{y}}$ & -4.1220 \\
\hline$\mu_{\mathrm{z}}$ & 0.0183 \\
\hline$\mu_{\text {total }}$ & 4.2457 \\
\hline
\end{tabular}

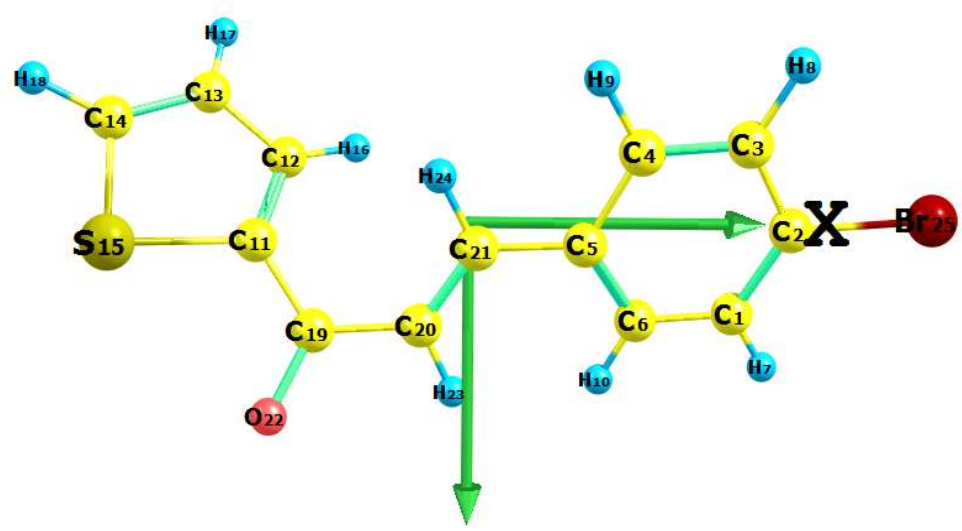

Fig-5: Dipole Moment of 3-(4-Bromophenyl)-1-(thiophene-2-yl) prop-2-en-1 one

\section{HOMO and LUMO Analysis}

HOMO and LUMO are very important roles of electron transfer are known as frontier molecular orbitals (FMOs). HOMO and LUMO ( $\pi$-acceptor) the energy gap was calculated. The HOMO and LUMO analysis have been measured by B3LYP/6-311G (d,p) basis set. The HOMO and LUMO of BPTP are given in Table-7 and Fig-6. The HOMO is presented at the thiophene ring and LUMO is presented at the chalcone moiety and the energy values are $-7.06370 \mathrm{eV}$ and $-0.58585 \mathrm{eV}$, correspondingly. BPTP molecule calculated energy gap is $-6.47785 \mathrm{eV} .{ }^{17}$ The physicochemical properties of conclusive charge transfer explain. The BPTP title compound using Koopmans theory and given in table; results of Koopmans theorem HOMO-LUMO and energy gap, the ionization potential (I)-ELUMO, electron affinity $(\mathrm{A})=-\mathrm{ELUMO}$, softness $(v)$ is $v=1 / \eta$, the global hardness $(\eta)$ is $\eta=(\mathrm{I}-\mathrm{A}) / 2$, Chemical potential $(\mu)$ is $\mu=-(\mathrm{I}+\mathrm{A}) / 2$, global electrophilicity $(\omega)$ is $\omega=\mu 2 / 2 \eta$ and Electronegativity $(\chi)$ is $\chi=(\mathrm{I}+\mathrm{A}) / 2{ }^{18}$

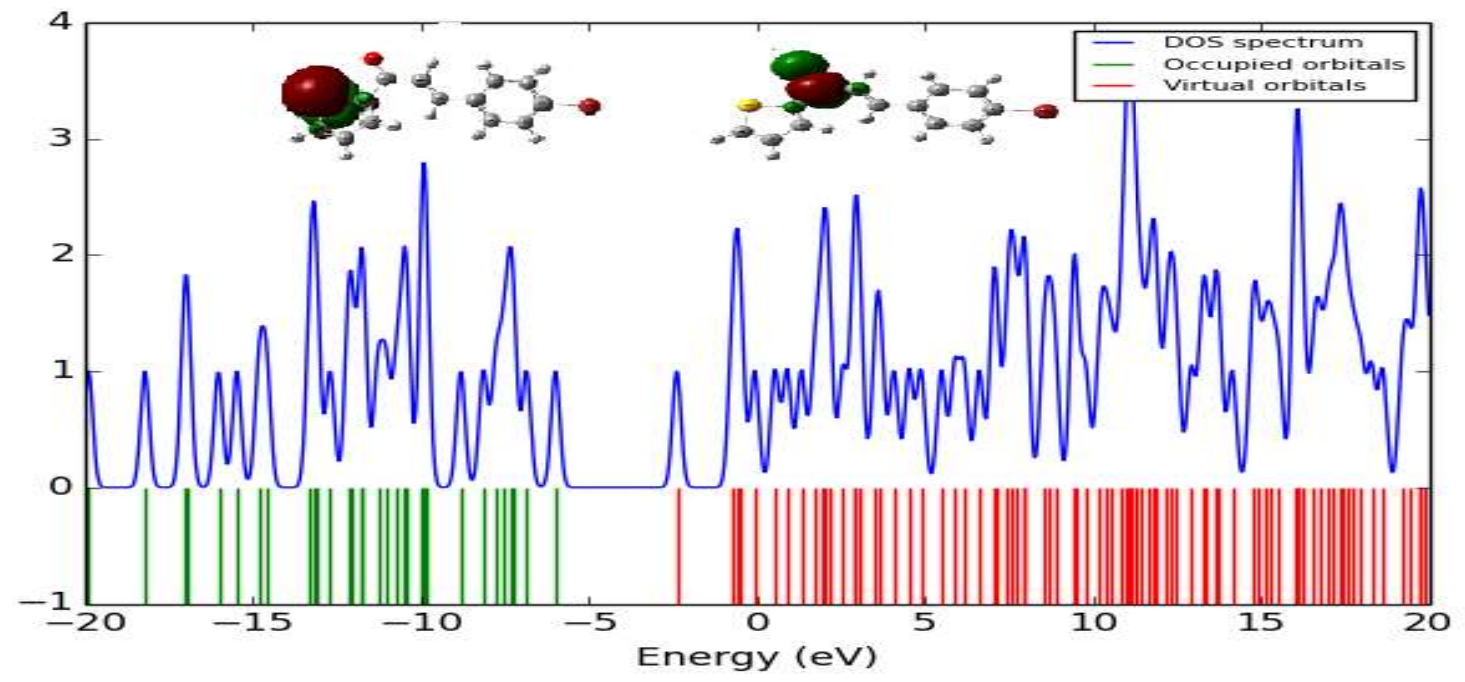

Fig -6: Density Functional Theory HOMO-LUMO of 3-(4-Bromophenyl)-1-(thiophen-2-yl) prop-2-en-1 one 
RASĀYAN J. Chem.

Vol. 12 | No. 4 |2149 - 2165| October - December | 2019

Table -7: HOMO and LUMO Calculated Values of 3-(4-Bromophenyl)-1-(thiophen-2-yl) prop-2-en-1 one by B3LYP/6-311G (d,p) Method.

\begin{tabular}{l|c}
\hline BASIS SET & B3LYP/6-311G(d,p) \\
\hline $\mathrm{E}_{\text {Homo }}(\mathrm{a} . \mathrm{u})$ & -7.0637 \\
\hline $\mathrm{E}_{\text {Lumo }}(\mathrm{a} . \mathrm{u})$ & -0.5858 \\
\hline Energy gap(a.u) & -6.4778 \\
\hline Ionization energy(I) & 7.0637 \\
\hline Electron affinity(A) & 0.5858 \\
\hline Global hardness $(\eta)$ & -3.8247 \\
\hline Chemical potential $(\mu)$ & -3.2389 \\
\hline Electro philicity index $(\omega)$ & 1.3714 \\
\hline Chemical softness(s) & -0.2614 \\
\hline
\end{tabular}

\section{Molecular Electrostatic Potential}

The molecular electrostatic potential (MEP) was mostly used. Its potential diagram is found in areas where it is possible. The simplest method was used to calculate the different connections on the MEP diagram. The reactive sites for nucleophiles and electrophilic attract of the MEP was measured B3LYP/6$311 \mathrm{G}(\mathrm{d}, \mathrm{p})$ basis set. The negative sites were presented in the carbonyl group and positive sites were presented in thiophenea moiety is given in Fig-7.
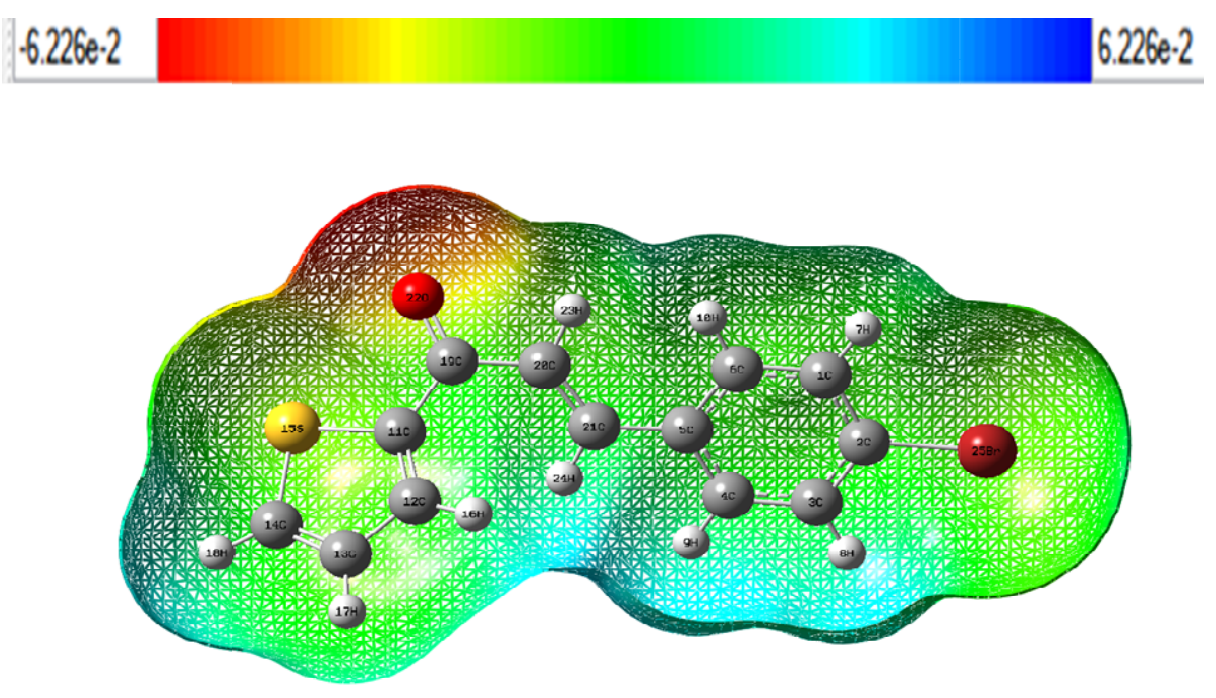

Fig-7: Molecular Electrostatic Potential (MEP) of 3-(4-Bromophenyl)-1-(thiophene-2-yl) prop-2-en-1 one

\section{Milliken Atomic Charges}

The charge evaluates the electronic structure charges under the atomic movement. It is connected straightforwardly to the chemical bonds shown in the title compound, its position, polarizability, electronic structure, dipole moment, and more effects of molecular systems, The Mullikan and natural charge sharing of the compound calculated on B3LYP /6-311G(d,p) technique are given in Table- 8 and Fig-8.The Mulliken charge distribution in the BPTP is found to be atoms C1,C6,C11,C12, S15,C19,C20,C21, and $\mathrm{Br} 25$ positive Natural charge and Mulliken charge possess definite charge in Mulliken $\mathrm{C} 1, \mathrm{C} 2, \mathrm{C} 3, \mathrm{C} 4, \mathrm{C} 5, \mathrm{C} 6, \mathrm{C} 11, \mathrm{C} 13, \mathrm{C} 14, \mathrm{C} 21, \mathrm{C} 22$ and $\mathrm{S} 15$ possess negative natural charge and Mulliken charge. ${ }^{19}$

\section{NBO Analysis}

The E (2) value displays are more related to the donor and acceptor BPTP molecular. The most definitive structure of the $\mathrm{C}=\mathrm{C}$ ring consists of electrons hyperconjugation connections with $\sigma$ and $\pi$. In the current debate $\pi$-character and $\sigma$ character play an important role. The hyperconjugation interactions $\pi(\mathrm{C} 12-$ 
RASĀYAN J. Chem.

Vol. 12 | No. 4 |2149 - 2165| October - December | 2019

$\mathrm{C} 13) \rightarrow \pi^{*}(\mathrm{C} 12-\mathrm{C} 17), \pi(\mathrm{C} 13-\mathrm{C} 14) \rightarrow \pi^{*}(\mathrm{C} 13-\mathrm{C} 14)$, transfer stabilization energy: 24.66 and $22.32 \mathrm{KJ} / \mathrm{mol}$ to the molecular arrangement. The lone pair of sulphur, bromine, and oxygen atom plays a good role in BPTP. The S1, O7, and Br18 atoms relocate the energy $24.78,15.42$ and $1.76 \mathrm{KJ} / \mathrm{mol}$ to $(\mathrm{C} 2-\mathrm{C} 3),(\mathrm{C} 2-$ C6) and $\mathrm{C} 15$ bonds, correspondingly. The extreme hyperconjugation E (2) energy of chalcone compare moiety during the inter-molecular interaction leads the molecular biological and medicinal applications. The bond $\sigma(\mathrm{S} 1-\mathrm{C} 2) 3.97 \mathrm{KJ} / \mathrm{mol}$ to $\sigma^{*}(\mathrm{~S} 1-\mathrm{C} 2)$ bond on comparing with energy transfer $3.97 \mathrm{KJ} / \mathrm{mol}$ from $\left.\pi(\mathrm{C} 4-\mathrm{C} 5) \rightarrow \pi^{*}(\mathrm{C} 4-\mathrm{C} 5) 15.31 \mathrm{KJ} / \mathrm{mol} \pi(\mathrm{C} 6-\mathrm{O} 7) \rightarrow \pi^{*}(\mathrm{C} 6-\mathrm{O} 7)\right) 7.79 \mathrm{KJ} / \mathrm{mol}$ given in Table- 9 .

\section{Molecular Docking Analysis}

Autodock 4.2 tools are used by molecular docking calculating binding mode and competence connector. The molecular analysis section of cancel cell (PDI: 8F8G) is used as a receptor and Autodock 4.2 package simulation. Autodock 4.2 tools are created using a protein system. Therefore, polar hydrogen bonds are provided. PDB is constructed $80 \mathrm{~A}^{\circ} \times 80 \mathrm{~A}^{\circ} \times 80 \mathrm{~A}^{\circ}$ using a structural analysis bonding and make use of the size of the grid to create energy. Cancer cell-related blocking $8 \mathrm{~F} 8 \mathrm{G}$ protein had good binding energy (7.68 stone $/ \mathrm{mol}$ ), which clearly illustrates the formation of hydrogen bonding protein is given Fig-9a. The pyMol package utilized for the visualizations of proton and target compound interactions. Discovery studio package 4.0 is used to detect the best bond in $2 \mathrm{D}$ is shown in Fig.-9b.

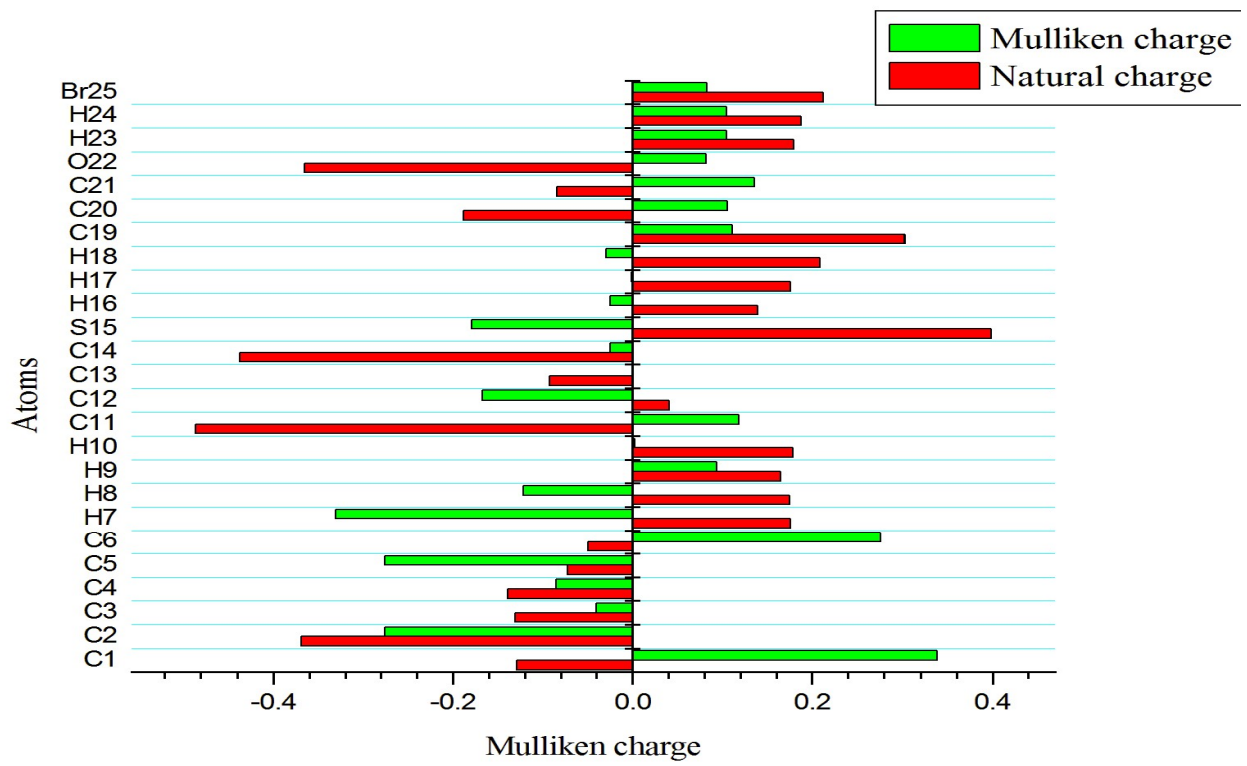

Fig-8: Milliken Charge Distribution of 3-(4-Bromophenyl)-1-(thiophen-2-yl) prop-2-en-1 one

Table-8: Mulliken Charges of 3-(4-bromophenyl)-1-(thiophen-2-yl) prop-2-en-1 one

\begin{tabular}{c|c|c}
\multicolumn{1}{l|}{ Atom } & Mulliken charge & Natural charge \\
\hline C1 & -0.128443 & 0.338669 \\
\hline C2 & -0.368945 & -0.275715 \\
\hline C3 & -0.130388 & -0.040173 \\
\hline C4 & -0.139122 & -0.084954 \\
\hline C5 & -0.072702 & -0.275627 \\
\hline C6 & -0.049014 & 0.27606 \\
\hline H7 & 0.175943 & -0.330222 \\
\hline H8 & 0.175004 & -0.121738 \\
\hline H9 & 0.165105 & 0.09335 \\
\hline H10 & 0.178777 & 0.002848 \\
\hline
\end{tabular}


RASĀYAN J. Chem.

Vol. 12 | No. 4 |2149 - 2165| October - December | 2019

\begin{tabular}{c|c|c}
\hline C11 & -0.486188 & 0.11868 \\
\hline C12 & 0.04107 & -0.167293 \\
\hline C13 & -0.092632 & -0.000628 \\
\hline C14 & -0.436983 & -0.024634 \\
\hline S15 & 0.398703 & -0.179176 \\
\hline H16 & 0.139821 & -0.025176 \\
\hline H17 & 0.17606 & -0.001525 \\
\hline H18 & 0.208638 & -0.029418 \\
\hline C19 & 0.302433 & 0.110706 \\
\hline C20 & -0.18797 & 0.105283 \\
\hline C21 & -0.084085 & 0.135985 \\
\hline O22 & -0.365313 & 0.081979 \\
\hline H23 & 0.179434 & 0.104858 \\
\hline H24 & 0.187959 & 0.104851 \\
\hline Br25 & 0.212837 & 0.083009 \\
\hline
\end{tabular}

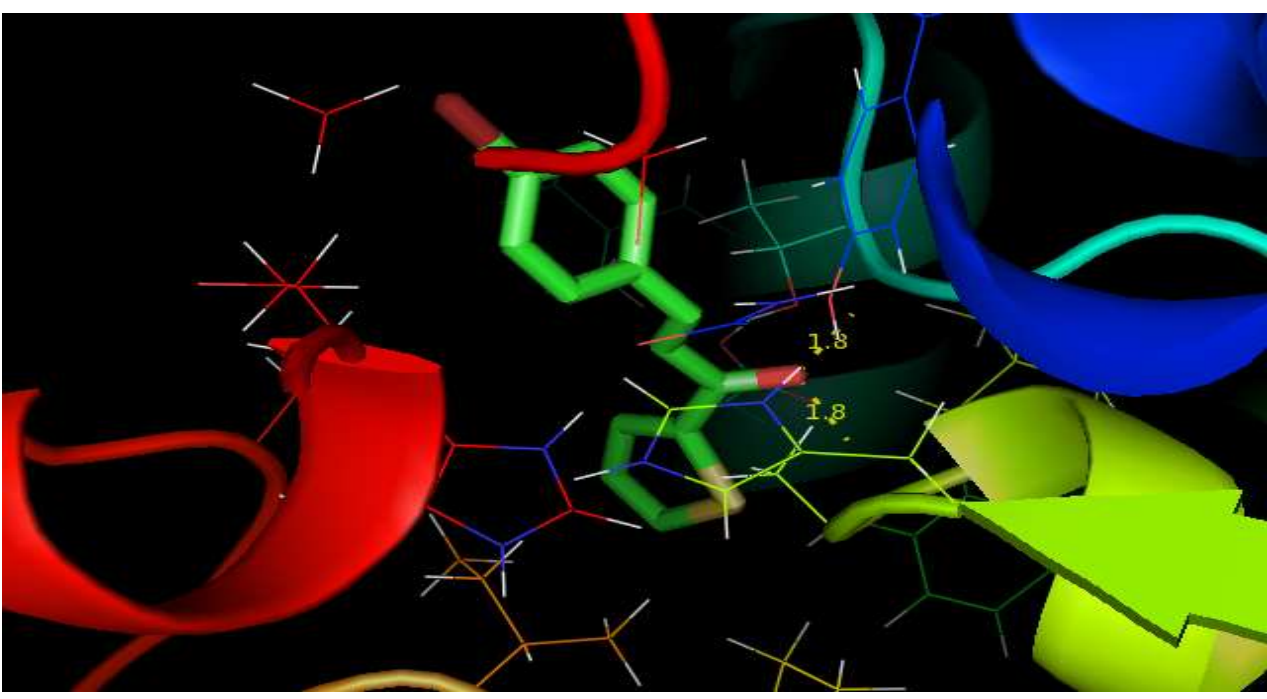

Fig. -9a: The docked pose of the 3-(4-bromophenyl)-1-(thiophen-2-yl) prop-2-en-1 one

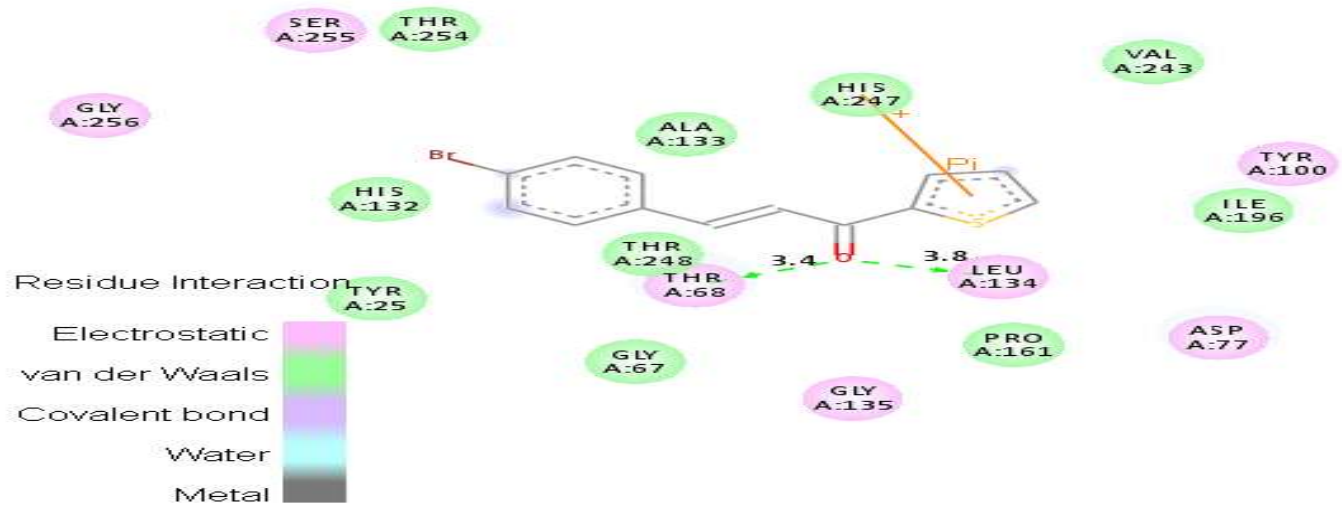

Fig.-9b: 2D the 3-(4-bromophenyl)-1-(thiophen-2-yl) prop-2-en-1 one 
RASĀYAN J. Chem.

Vol. 12 | No. 4 |2149 - 2165| October - December | 2019

The length of the binding obtained at the end of the test is hydrogen bonding with THR A: 6.8 (threonine) interactive at 3.4 and LEUA:134 (teucine) interactive at 3.8 releases both Amino acids. ${ }^{20}$ The final result is confirmed that the target compound is one of the best solutions for cancer treatment the results are shown in Table $-10 .^{21}$

Table-9: Second-Order Perturbation Theory Analysis of Fock Matrix in NBO Basis Corresponding to the Intermolecular Bonds of the BPTP Compound

\begin{tabular}{c|c|c|c|c|c|c|c|c}
\hline Donar & Type & ED/e & Acceptor & Type & ED/e & Kcal /mol & $\begin{array}{c}\text { Ej-Ei } \\
\text { a.u }\end{array}$ & $\begin{array}{c}\text { F(I,j) } \\
\text { a.u }\end{array}$ \\
\hline S1-C2 & $\sigma$ & 1.9701 & S1-C2 & $\sigma^{*}$ & 0.3422 & 03.97 & 1.10 & 0.059 \\
\hline S1-C5 & $\sigma$ & 1.9812 & S1-C5 & $\sigma^{*}$ & 0.0177 & 04.16 & 1.10 & 0.060 \\
\hline C2-C3 & $\pi$ & 1.7846 & C2-C3 & $\sigma^{*}$ & 0.3593 & 24.05 & 0.32 & 0.079 \\
\hline C2-C6 & $\sigma$ & 1.9729 & C2-C6 & $\sigma^{*}$ & 0.0542 & 06.10 & 1.33 & 0.00 \\
\hline C3-C4 & $\sigma$ & 1.9766 & C3-C4 & $\sigma^{*}$ & 0.01455 & 04.31 & 1.29 & 0.007 \\
\hline C4-C5 & $\pi$ & 1.8368 & C4-C5 & $\pi$ & 0.3116 & 15.31 & 0.30 & 0.064 \\
\hline C6-O7 & $\pi$ & 1.9426 & C6-O7 & $\pi$ & 0.2684 & 07.79 & 0.41 & 0.051 \\
\hline C8-C10 & $\sigma$ & 1.8409 & C6-C10 & $\pi$ & 0.0687 & 30.36 & 0.31 & 0.090 \\
\hline C10-C12 & $\sigma$ & 1.9657 & C10-C12 & $\sigma$ & 0.0276 & 5.45 & 1.40 & 0.078 \\
\hline C12-C13 & $\sigma$ & 1.9527 & C12-C13 & $\sigma$ & 0.0340 & 5.31 & 1.26 & 0.073 \\
\hline C12-C17 & $\pi$ & 1.6318 & C12-C17 & $\pi$ & 0.3638 & 24.66 & 0.21 & 0.073 \\
\hline C13-C14 & $\pi$ & 1.6728 & C13-C14 & $\pi$ & 0.3208 & 22.32 & 0.29 & 0.072 \\
\hline C15- & $\sigma$ & 1.9832 & C15- Br18 & $\sigma$ & 0.0331 & 02.90 & 1.25 & 0.054 \\
\hline Br18 & & & & & & & & \\
\hline $\mathrm{Lp}(2)$ & $\mathrm{S} 1$ & & & $\pi$ & 1.5755 & & & \\
\hline $\mathrm{Lp}(2)$ & $\mathrm{O} 7$ & & & $\pi$ & 1.8913 & & & \\
\hline $\mathrm{Lp}(2)$ & $\mathrm{Br} 18$ & & & $\pi$ & 1.9753 & & & \\
\hline
\end{tabular}

Table -10: Estimated Free Energy, Electrostatic Energy, Inhibition Constant, and Total Molecular Energy Obtained From Ligand-Protein Interactions Between the Target Receptor 8F8G and BPTP.

\begin{tabular}{c|c|c|c|c|c|c|c|c}
\hline S.No. & $\begin{array}{c}\text { Binding } \\
\text { Energy } \\
(\mathrm{Kcal} / \\
\mathrm{Mol})\end{array}$ & $\begin{array}{c}\text { Ligand } \\
\text { Efficiency }\end{array}$ & $\begin{array}{c}\text { Inhibition } \\
\text { Constant } \\
\mathrm{Ki}(\mu \mathrm{M})\end{array}$ & $\begin{array}{c}\text { Intermolecular } \\
\text { Energy } \\
(\mathrm{Kcal} / \mathrm{Mol})\end{array}$ & $\begin{array}{c}\text { Vdw + H- } \\
\text { Bonding }+ \\
\text { Desolv. } \\
\text { Energy } \\
\text { Kcal/Mol })\end{array}$ & $\begin{array}{c}\text { Electrostat } \\
\text { ic Energy } \\
(\text { Kcal/Mol })\end{array}$ & $\begin{array}{c}\text { Total } \\
\text { Internal } \\
\text { Energy }\end{array}$ & $\begin{array}{c}\text { Torsion } \\
\text { Energy } \\
(\text { Kcal/ } \\
\text { Mol })\end{array}$ \\
\hline 1 & -7.68 & -0.48 & 2.33 & 8.58 & -8.48 & -0.1 & -0.35 & 0.89 \\
\hline 2 & -7.09 & -0.44 & 6.41 & -7.98 & -7.90 & -0.08 & -0.36 & 0.89 \\
\hline 3 & -7.08 & -0.44 & 6.42 & -7.98 & -7.89 & 0.08 & -3.36 & 0.89 \\
\hline 4 & -7.06 & -0.44 & 6.66 & -7.96 & -7.89 & -0.07 & -3.39 & 0.89 \\
\hline 5 & -6.88 & -0.43 & 9.07 & -7.77 & -7.64 & -0.13 & -0.36 & 0.89 \\
\hline 6 & -6.79 & -0.42 & 10.55 & -7.68 & -7.52 & -0.17 & -0.38 & 0.89 \\
\hline 7 & -6.53 & -0.41 & 16.22 & -7.43 & -7.22 & -0.21 & -0.43 & 0.89 \\
\hline 8 & -6.47 & -0.40 & 18.24 & -7.36 & -7.36 & 0.0 & -0.41 & 0.89 \\
\hline 9 & -6.38 & -0.4 & 21.1 & -7.27 & -7.15 & -0.13 & 0.38 & 0.89 \\
\hline 10 & -6.31 & -0.39 & 23.72 & -7.20 & -7.06 & -0.14 & 0.45 & 0.89 \\
\hline
\end{tabular}

\section{CONCLUSION}

The synthesized molecule 3-(4-bromophenyl)-1-(thiophene-2-yl) prop-2-en-1one (BPTP) was characterized by using several analytical methods such as FT-IR, and NMR. The B3LYP/6-311G basis 3-(4-BROMOPHENYL)-1-(THIOPHEN-2-YL) PROP-2-EN-1 ONE 
RASĀYAN J. Chem.

Vol. 12 | No. 4 |2149 - 2165| October - December | 2019

set was adopted for target compound optimization. The experimentally recorded FT-IR and FT-Raman spectrum was remarkable in the agreement with theoretical one. Nuclear magnetic resonance spectroscopy was confirmed the structure of the BPTP; proton and carbon NMR chemical shifts were determined and compared with the experimental values. Natural bond orbital study stabilizes that the intermolecular charge relocates formed between $\pi(\mathrm{C} 6-\mathrm{O} 7) \rightarrow \pi^{*}(\mathrm{C} 6-\mathrm{O} 7)$. The experimental UV-Visible absorption spectrum was good agreement within the BPTP theoretical one. The FMOs energy gap given in the compound exhibit the charge transfer in takes place in the compound. The molecular electrostatic potential map utilized to identify the nucleophilic and electrophilic attacks nature. The synthesized compound atomic theory was also been made with NBO and Milliken, MEP results and Fukui indices.

\section{ACKNOWLEDGMENT}

The authors are thankful to SAIF, IIT, and Madras for providing Spectral measurements.

\section{REFERENCES}

1. R. Shivaraj Maidur, P.S. Patil, A. Ekbote, T.S. Chia, and C.K. Quah, Spectrochimica Acta Part A: Molecular and Biomolecular Spectroscopy, 184, 342(2017), DOI: 10.1016/j.saa.2017.05.015

2. S. Tarchouna, I. Chaabane, and A. Ben Rahaiem, Physica E: Low-dimensional Systems and Nanostructures 83, 186(2016), DOI: 10.1016/j.physe.2016.05.021

3. F. Weinhold, J. Gauss view, Am. Chem. Phys. Soc, 102, 7211(1980).

4. E. Reed, A. Alan Larry Curtiss, and Frank Weinhold, Chemical Reviews, 88, 899(1988), DOI: 10.1021/cr00088a005

5. S. Bharanidharan, H. Saleem, S. Subaschchandrabose, M. Suresh and N. Ramesh Babu, Arch. Chem. Res., 1, 2(2017), DOI: 10.21767/2572-4657.100007

6. A. Nathiya, H. Saleem, S. Bharanidharan and M. Suresh, International Journal of Advanced Chemistry, 6, 53(2018), DOI: 10.14419/ijac.v6i1.9046

7. P.K. Ranjith, Y. Sheena Mary, C. Yohannan Panicker, P.L. Anto, Stevan Armaković, Sanja J. Armaković, Robert Musiol, Josef Jampilek, C. Van Alsenoy, Journal of Molecular Structure, 1, 1135(2017), DOI:10.1016/j.molstruc.2017.01.045

8. V.Vijayakumar, A. Prabakaran, N. Radhakrishnan, S. Muthu, C. Rameshkumar, and E.I. Paulraj, Journal of Molecular Structure, 1179, 325(2018), DOI:10.1016/j.molstruc.2018.11.018

9. J. Dhevaraj, S. Vembu, S. Pazhamalai and M. Gopalakrishnan, Oriental Journal of Chemistry, 35, 577(2019), DOI:10.13005/ojc/350211

10. T. S. Xavier, Naghmana Rashid, and I. Hubert Joe, Spectrochimica Acta Part A: Molecular and Biomolecular Spectroscopy, 78, 319(2011), DOI: 10.1016/j.saa.2010.10.013

11. N. Issaoui, H. Ghalla, F.Bardak, M. Karabacak, N.A. Dlala, H.T. Flakus, Journal of Molecular Structure, 1130, 659(2017), DOI:10.1016/j.molstruc.2016.11.019

12. G. Shakila and H. Saleem, World Scientific News, 61(2), 150(2017).

13. K. Senthil1, K. Elangovan, A. Senthil and G. Vinitha, Rasayan J. Chem., 12, 1262(2019), DOI: 10.31788/RJC.2019.1235174

14. S. John Pradeepa, D. Tamilvendan, M.S. Boobalan, and N. Sundaraganesan, Journal of Molecular Structure, 1112, 33(2016), DOI: 10.1016/j.molstruc.2016.01.026

15. R. Govindhan, and B. Karthikeyan, Journal of Physics and Chemistry of Solids, 111, 123(2017), DOI:10.1016/j.jpcs.2017.07.025

16. P.Rajamani, N. Sundaraganesan, V. Vijayakumar, M.S Boobalan, and M. Jeeva, J. of Molecular Structure, 1197, 417(2019), DOI:10.1016/j.molstruc.2019.07.040

17. A. Mohamed Ibrahim and S. Arunachalam, Rasayan J. Chem., 12, 1219(2019), DOI: 10.31788/Rjc.2019.1235172

18. S. Nithya, B. Chandar Shekar, K. R. Aranganayagam and K. Boopathi, Rasayan J. Chem., 12, 1399(2019), DOI: 10.31788/RJC.2019.1235307

19. A. Alphonsa, C.Therasa, S. Loganathan, Athavan Alias Anand, and S. Kabilan, Journal of Molecular Structure, 1130, 1018(2017), DOI:10.1016/j.molstruc.2016.10.005

20. B. Geetha, G. Swarnalatha and G.V. Subba Reddy, Rasayan J. Chem., 12, 63(2019), DOI: 10.31788/RJC.2019.1235165a 
RASĀYAN J. Chem.

Vol. 12 | No. 4 |2149 - 2165| October - December | 2019

21. V. Veeraragavan, N. Radhakrishnan and C. Ramesh Kumar, International Conference on Smart Technologies and Management for Computing, Communication, Controls, Energy and Materials, 4, 513, (2017), DOI:10.1109/ICSTM.2017.8089213.

[RJC-5436/2019] 